\title{
Pleomorphic Hyalinizing Angiectatic Tumor Arising in the Hand: A Case Report
}

\author{
Patrick M. Kane', Michael P. Gaspar', Benjamin B. Whiting', and Randall W. Culp'
}

\begin{abstract}
Background: Background: Pleomorphic hyalinizing angiectatic tumors (PHATs) are extremely rare, non-metastasizing tumors of uncertain origin that are typically seen in the lower extremities. To date, it is estimated that less than 100 cases have been reported worldwide since first described in 1996. Methods: The case of a 35-year-old male with a several-year history of a dorsal hand mass is presented. Although the patient was initially asymptomatic, in the months prior to presentation, the patient complained of pain with power grasp and direct pressure over the mass. The patient underwent uncomplicated surgical excision, during which the mass was noted to be adherent to the underlying extensor tendons. Results: Immunopathology confirmed the mass to be PHAT. We believe this is the first documented case of this rare tumor occurring in the hand. Conclusions: History and epidemiology of PHAT are reviewed. Then, in the context of the presented case, pre-operative evaluation, surgical management, pathologic findings and post-operative follow-up are all discussed.
\end{abstract}

Keywords: pleomorphic hyalinizing angiectatic tumor, mass, hand

\section{Background}

Pleomorphic hyalinizing angiectatic tumor (PHAT) is a rare soft-tissue neoplasm which was first described by Smith et al in a case series of 14 patients. ${ }^{8}$ To date, it is estimated that less than 100 cases have been reported worldwide. ${ }^{2}$ PHATs are non-metastasizing soft-tissue tumors, categorized as "tumors of uncertain differentiation" based on the 2013 World Health Organization classification. ${ }^{11}$ They most frequently occur within the superficial subcutaneous tissues and in muscles. ${ }^{3,5}$ Case reports describe patients as young as 10 , and as old as 83 years of age, although most patients presented in between their fourth and sixth decades. However, given the relatively few number of cases presented, exact epidemiologic patient characteristics are debated, and have potential to evolve as more cases are reported. ${ }^{5}$

\section{Case Report}

A 35-year-old, right-hand-dominant male corrections officer presented with a chief complaint of a mass located on the dorsum of his right hand. He stated that the mass had been present for several years and he felt it had gradually increased in size over the previous 2 to 3 months. In addition, the mass had gradually become painful with power grasp and direct pressure over the area within those recent months. Past medical, family, and social history were all non-contributory, and the patient did not take any medications. He denied any prior history of trauma or surgery to his right upper extremity.
On physical exam, there was a palpable mass present over the dorsum of the ulnar aspect of his right hand measuring approximately $3 \times 4.5 \mathrm{~cm}$. It was noted to be mobile and soft, with no appreciable bruit. Range of motion of all wrist and finger joints was within normal limits. Prior magnetic resonance imaging (MRI) was consistent with a lowflow hemangioma (Figures 1a and 1b).

The patient underwent surgical excision, where the mass was noted to be adherent to the underlying extensor tendons at the level of the fourth and fifth metacarpals. After excision of the mass in its entirety, the specimen was sent to pathology for analysis and the skin was closed (see Figure 2).

\section{Pathology Report}

The specimen measured $4.6 \times 2.8 \times 1.8 \mathrm{~cm}$ in size. On gross inspection, it was noted to have a tan-gray/pink color and was partially encapsulated with an irregular shape. Sectioning the mass revealed multiple hemorrhagic nodules measuring between 0.8 and $2.3 \mathrm{~cm}$ in largest dimension. Microscopically, the tumor was composed of pleomorphic multinucleated neoplastic cells, with hemosiderin deposition predominantly at the tumor periphery. Dilated blood

\footnotetext{
'The Philadelphia Hand Center, Philadelphia, PA, USA
}

\section{Corresponding Author:}

Michael P. Gaspar, The Philadelphia Hand Center, The Franklin Suite GI I4, 834 Chestnut Street, Philadelphia, PA 19107, USA.

Email: michaelpgaspar@gmail.com 

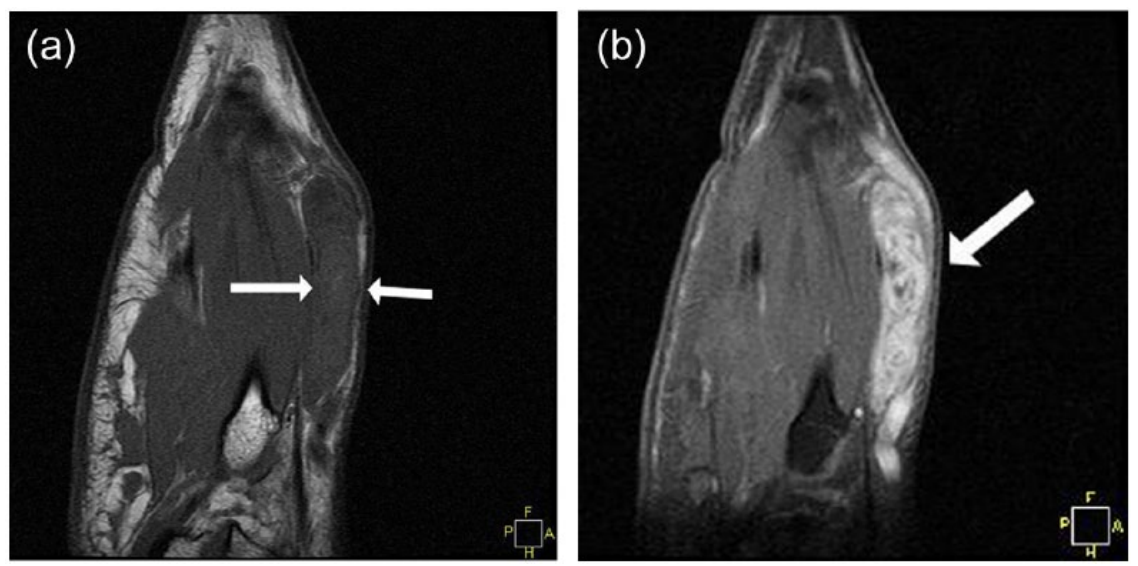

Figure I. Magnetic resonance imaging (MRI) findings: (a) TI-weighted sagittal image reveals the dorso-ulnar hand mass with signal isointense to that of skeletal muscle (between arrows) and (b) Fat-saturated gadolinium-enhanced sagittal imaging demonstrates heterogeneous enhancement of the mass.

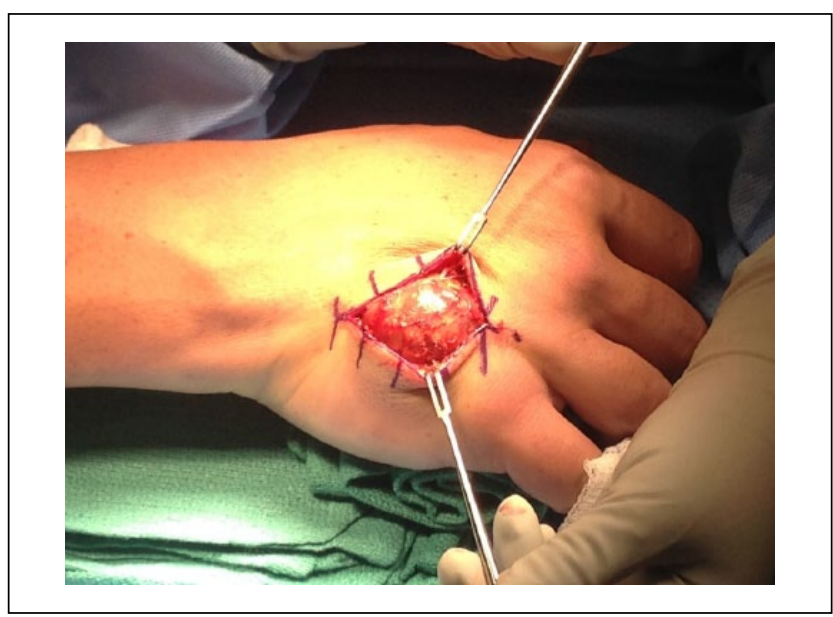

Figure 2. Intraoperative photograph showing the tumor in situ, just prior to excision.

vessels with a peripheral hyalinization and luminal fibrin were also present (Figure 3). Mitotic activity was low, measured by a proliferation marker (Ki-67) to be approximately $10 \%$. Immunohistochemical staining was positive for CD99 and negative for alpha smooth muscle cell actin (SMA) and desmin, and the diagnosis of PHAT was made.

\section{Discussion}

PHATs are rare, non-metastasizing tumors of uncertain lineage, first reported in 1996. On gross appearance, excised masses have been described as white-tan to yellowish color, and punctate hemorrhage and vessel thrombosis are often seen ${ }^{3,8}$ Histologically, the tumor cells are similar to malignant fibrous histiocytoma but have low-mitotic

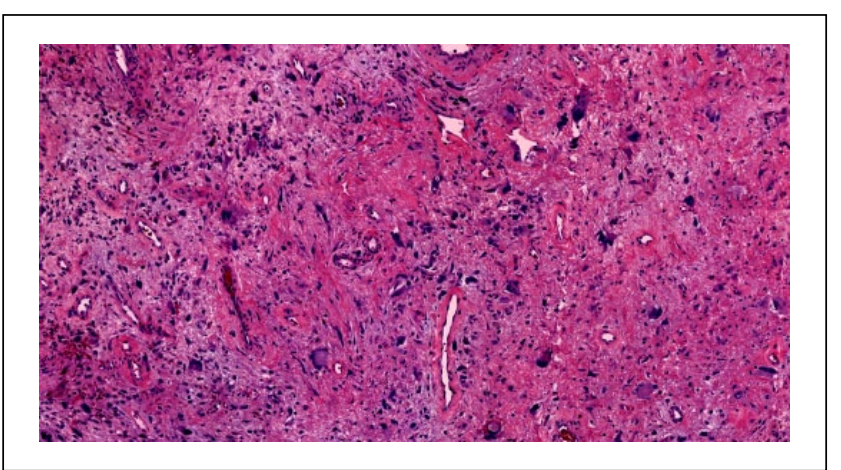

Figure 3. Histopathological findings under high-power magnification reveal hemosiderin deposition at the periphery and dilated vessels with the characteristic peripheral hyalinization (hematoxylin and eosin stain $\times 100$ ).

figure, and can be characterized by clusters of ectatic, fibrin-lined, thin-walled vessels, surrounded by pleomorphic neoplastic stoma with variable inflammatory cells. ${ }^{3,8}$ Immunohistochemical staining typically shows cells to be positive for CD34 and vimentin and may be positive for CD99 and vascular endothelial growth factor (VEGF), although they are usually negative for S-100, human melanoma black 45 (HMB45), and actin.

In the largest case series to date, 31 of the 41 cases $(75.6 \%)$ occurred in the lower extremity versus only 4 (9.8\%) in the upper extremity. ${ }^{3}$ In the 18 patients with follow-up, 6 patients developed recurrence. Local recurrence rates have been reported between $33 \%$ and $50 \%{ }^{10}$ All tumors that have recurred were initially treated with marginal excision, while no tumors have recurred after wide excision. ${ }^{3}$ Generally, recurrences are not destructive, although one case report described an aggressive recurrence 


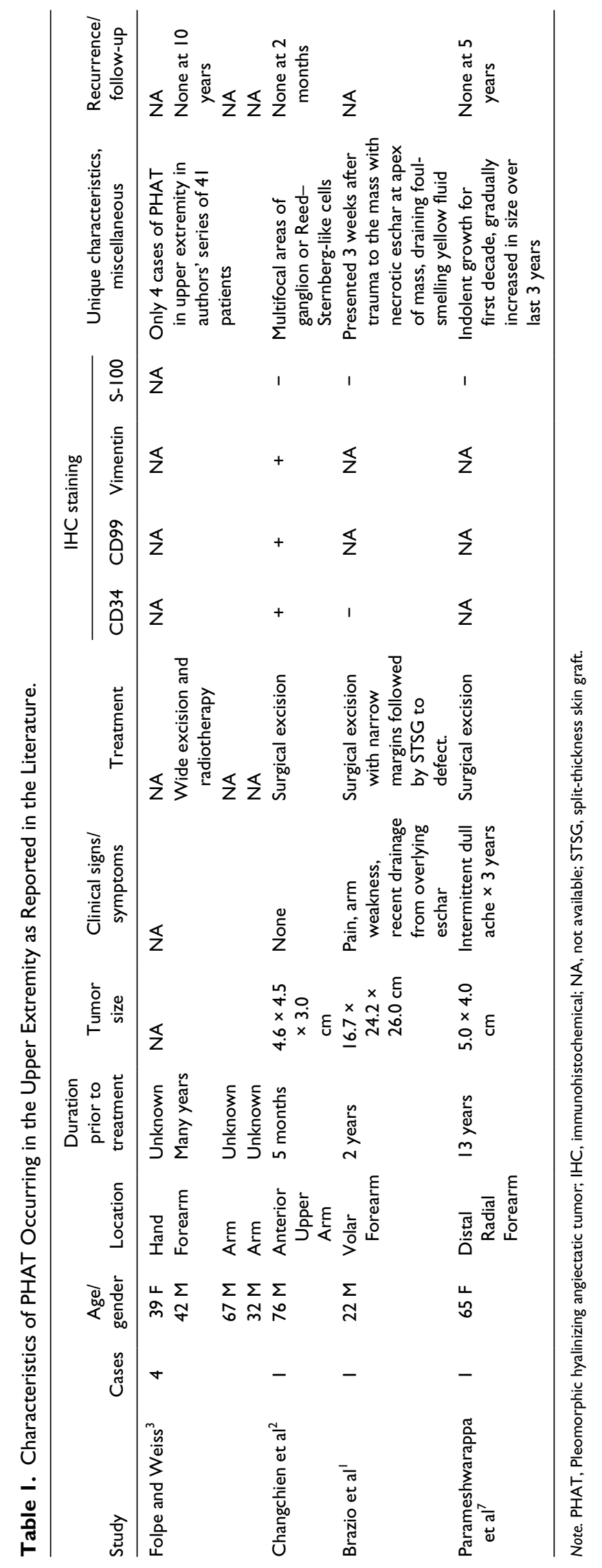


requiring amputation. ${ }^{3}$ In rare cases, the tumors have recurred with sarcomatous characteristics., ${ }^{3,4,6}$

To date, published reports of PHAT have primarily focused on pathological findings of disease, with only one study describing the imaging characteristics of this tumor. ${ }^{9}$ The authors of that study concluded that PHAT should be included on the differential diagnosis when encountering an enhancing, subcutaneous tumor with ill-defined margins, particularly in the extremity. This is the first described case with imaging of a PHAT tumor described in the hand.

Due to the potential for local recurrence, surgical excision with a tumor-free margin is the preferred treatment for PHAT. ${ }^{5}$ This could pose a particularly challenging problem in the hand or wrist, where wide excision of tumors has potential to jeopardize critical neurovascular and/or musculotendinous structures and thus compromise function. In the presented case, however, the tumor was freed from the underlying extensor tendons uneventfully. Table 1 outlines the key features of the various reports of upper-extremity PHAT in recent literature.

With regard to adjuvant therapy, few investigators have advocated for low-dose radiotherapy in cases of known incomplete resection to avoid recurrence; however, there is presently no data to support such a regimen. ${ }^{10}$ Despite its apparent lack of potential for metastases, the importance of adequate surgical removal of the tumor is illustrated by the potential for aggressive or sarcomatous recurrences. ${ }^{3,4,6,8}$ Further studies are required to delineate the clinical course and long-term outcomes associated with this condition, as well as the efficacy of adjuvant modalities when excision is inadequate. Although we recognize the importance of more extensive clinical followup, we feel the novelty of this case of PHAT occurring in the hand warrants report and increases awareness of the characteristics and treatment pearls for this extremely rare tumor, particularly for hand surgeons.

\section{Ethical Approval}

This study was approved by our institutional review board.

\section{Statement of Human and Animal Rights}

All study procedures were followed in accordance with the ethical standards of the responsible committee on human experimentation (institutional and national) and with the Helsinki Declaration of 1975, as revised in 2000 and 2008.

\section{Statement of Informed Consent}

Informed consent was obtained from the patient included within the study.

\section{Declaration of Conflicting Interests}

The author(s) declared no potential conflicts of interest with respect to the research, authorship, and/or publication of this article.

\section{Funding}

The author(s) received no financial support for the research, authorship, and/or publication of this article.

\section{References}

1. Brazio PS, Morrison AL, Oh M, Goldberg N, Boutros $\mathrm{CN}$. Large pleomorphic hyalinizing angiectatic tumor of the forearm: a multidisciplinary perspective [published online ahead of print June 4, 2015]. Surgery. doi:10.1016/j. surg.2015.04.033.

2. Changchien YC, Bocskai P, Kovács I, et al. Pleomorphic hyalinizing angiectatic tumor of soft parts: case report with unusual ganglion-like cells and review of the literature. Pathol Res Pract. 2014;210(12):1146-1151.

3. Folpe AL, Weiss SW. Pleomorphic hyalinizing angiectatic tumor: analysis of 41 cases supporting evolution from a distinctive precursor lesion. Am J Surg Pathol. 2004;28: 1417-1425.

4. Kazakov DV, Pavlovsky M, Mukensnabl P, et al. Pleomorphic hyalinizing angiectatic tumor with a sarcomatous component recurring as high-grade myxofibrosarcoma. Pathol Int. 2007;57:281-284.

5. Lee JC, Jiang XY, Karpinski RH, et al. Pleomorphic hyalinizing angiectatic tumor of soft parts. Surgery. 2005;137:119-121.

6. Mitsuhashi T, Barr RJ, Machtinger LA, et al. Primary cutaneous myxofibrosarcoma mimicking pleomorphic hyalinizing angiectatic tumor (PHAT): a potential diagnostic pitfall. $\mathrm{Am} \mathrm{J}$ Dermatopathol. 2005;27:322-326.

7. Parameshwarappa S, Rodrigues G, Nagpal N, Rao L. Pleomorphic hyalinizing angiectatic tumor of soft parts involving the upper limb. Indian J Surg. 2010;72(3): 263-264.

8. Smith ME, Fisher C, Weiss SW. Pleomorphic hyalinizing angiectatic tumor of soft parts. A low-grade neoplasm resembling neurilemoma. Am J Surg Pathol. 1996;20:21-29.

9. Subhawong TK, Subhawong AP, Montgomery EA, et al. Pleomorphic hyalinizing angiectatic tumor: imaging findings. Skeletal Radiol. 2012;41:1621-1626.

10. Suzuki K, Yasuda T, Hori T, et al. Pleomorphic hyalinizing angiectatic tumor arising in the thigh: a case report. Oncol Lett. 2014;7:1249-1252.

11. Weiss SW, Dei Tos AP. Pleomorphic hyalinizing angiectatic tumour of soft parts, p. 200. In: Fletcher CDM, Bridge JA, Hogendoorn PCW, Mertens F, eds. WHO Classification of Tumours of Soft Tissue and Bone. Lyon, France: International Agency for Research on Cancer Press; 2013. 